\title{
A preliminary study on mycobiota and ochratoxin a contamination in commercial palm dates (Phoenix dactylifera)
}

\author{
Iva Nikolchina ${ }^{1} \cdot$ Paula Rodrigues ${ }^{1}$ (D)
}

Received: 6 November 2020 / Revised: 20 April 2021 / Accepted: 12 May 2021 / Published online: 21 May 2021

(c) Society for Mycotoxin (Research Gesellschaft für Mykotoxinforschung e.V.) and Springer-Verlag GmbH Germany, part of Springer Nature 2021

\begin{abstract}
The occurrence of mycotoxins and mycotoxigenic fungi in palm dates has not been thoroughly documented. The aims of the present study were to identify the mycobiota present in commercial date samples $(n=19)$, to determine the ability of the isolated fungi to produce mycotoxins, and to determine and quantify the presence of OTA in date fruits. The majority of products originated from Tunisia $(\mathrm{n}=14)$ and Algeria $(\mathrm{n}=3)$. The dominant fungal species were Aspergillus niger, Aspergillus tubingensis and Aspergillus flavus which were most frequently found in premium quality and organic produce, produced without chemical preservatives. OTA was found in only one sample at a concentration of $0.75 \mu \mathrm{g} / \mathrm{kg}$, as determined by HPLC with fluorescence detection. Although this preliminary study did not find elevated levels of OTA, its presence in one out of 19 samples indicates that palm dates for human consumption require continuous and stringent control, in order to prevent contaminated produce from entering the market.
\end{abstract}

Keywords Dried fruits $\cdot$ Mycotoxins $\cdot$ Food safety $\cdot$ Aspergillus $\cdot$ Penicillium

\section{Introduction}

Dried fruits are currently important foods throughout the world, as they are sources of diverse essential nutrients with potential functional properties. Fruits such as dates, prunes, apricots, figs and raisins are of major significance socially and economically for the Mediterranean populations (Ozer et al. 2012). The fruits of the date palm, in particular, contain a high percentage of sugars (44-88\%), protein $(2.3-5.6 \%)$ and dietary fibre $(6.4-11.5 \%)$, high diversity of salts and minerals and low fat (0.2-0.5\%) (Al-Shahib and Marshall 2003). Dates are also a good source of vitamins, mostly A and group B (Zaid and de Wet 2002). Dates are mainly produced in the desert of Southwest Asia and North Africa, but they are marketed worldwide as a high-value fruit crop, accounting for almost half of the 2 million tonnes of dried fruits traded worldwide (Calcagni 2018). In Europe, the Mediterranean countries France, Italy and Spain are the most important consumers of dried dates, with a yearly

Paula Rodrigues

prodrigues@ipb.pt

1 Centro de Investigação de Montanha (CIMO), Instituto Politécnico de Bragança, Campus de Santa Apolónia, 5300-253 Bragança, Portugal intake per capita of 140 to $340 \mathrm{~g}$ (FAOSTAT 2021). In Portugal, it is estimated that $17 \%$ of the Portuguese population consumes dried dates regularly (Ribeiro and Fernandes 2017), with an estimated intake of around $50 \mathrm{~g} /$ capita/year in 2017, but a two-fold increase was observed from 2017 to 2018 (FAOSTAT 2021). Date consumers are no longer limited to traditional ethnic population but are increasing among younger consumers (millennials) that follow healthy living trends (CBI 2020).

Dates mature through three stages during which colour, flavour, texture and microbial profile evolve (Shenasi et al. 2002a): Kimri, an inedible green fruit; then Rutab, a soft brown stage; and later Tamr, with dark brown colouration acquired after harvest and drying (Shenasi et al. 2002a). Throughout these stages, microbial counts also change; they are high at the Kimri stage, increase at the Rutab stage, and then significantly decrease at the final dried Tamr stage. Throughout all the three maturation stages, it is also possible that fungi develop and produce mycotoxins (Shenasi et al. 2002a, b). Given that these fruits are marketed not only as dried fruits for direct consumption but are also intensively incorporated in many food applications (CBI 2020), they could represent an important human dietary source of mycotoxins. 
According to previous surveys, it is noticeable that dried fruits are generally susceptible to fungal contamination and growth, and consequent mycotoxin production.In dried fruits, mycotoxin contamination has been mostly studied on aflatoxins (AFs) in dried figs, and on ochratoxin A (OTA) in dried vine fruits (e.g. Aksoy et al. 2007; Bircan and Koç 2012; Heperkan et al. 2012; Heshmati et al. 2017; Iamanaka et al. 2007), but mycotoxin contamination in dried dates has been poorly studied (Azaiez et al. 2015; Gherbawy et al. 2012; Ozer et al. 2012; Quaglia et al. 2020; Rahimi and Shakerian 2013;). Although the EU regulation 1881/2006 (EC 2006a) does not include OTA maximum admissible levels for dried palm dates, the latest EFSA report on OTA risk assessment reports dried fruits (including dates) among the most important contributors to the chronic dietary exposure of European populations to OTA, mostly in children and toddlers, after meat products, cheese and grain products (EFSA 2020). As a matter of consequence, palm dates should be given extra attention in the global food safety and security context, as they can be regarded not only as an increasing food in healthy diets but also as a good nutrient source able to supply the increased food demand expected for the years to come.

It is thus necessary to acknowledge the importance of toxigenic fungi in palm dates and their ability to produce mycotoxins, in particular OTA, and the level of contamination of commercial dates. This knowledge will be critical to establish a risk assessment as well as to develop preventive or corrective measures in the date chains, especially during drying and storage.

In this scenario, the main objectives of this study were to identify the mycobiota present in date samples commercialized in Portugal, to determine the ability of the isolated fungi to produce OTA and to determine and quantify the presence of OTA in the samples.

\section{Methods and materials}

\section{Sampling plan}

Nineteen samples of dried date fruits (200 g to $250 \mathrm{~g}$ ) representative of all types of dates available in the region were purchased in different markets in Portugal between April and August 2018. Samples characteristics are shown in Table S1.

\section{Fungal isolation from date samples}

In order to have a full understanding of the diversity of the native fungal species and to increase the sensitivity of fungi detection and isolation, two different methods of plating were used: serial dilutions and direct plating. Fruits were randomly taken from the package, cut in small pieces (without surface disinfection) and mixed. For the serial dilution method, $10 \mathrm{~g}$ were homogenized for $1 \mathrm{~min}$ with $90 \mathrm{~mL}$ of peptone water and further serially diluted. One hundred microlitres from each dilution were spread (in duplicate) in 9-cm Petri dishes containing $20 \mathrm{~mL}$ of Dichloran rose Bengal chloramphenicol (DRBC, Liofilchem). For the direct plating method, fruit pieces $(10 \mathrm{~g})$ were placed directly onto the DRBC in 9-cm Petri dishes (in duplicate). Plates were incubated in the dark, at $25^{\circ} \mathrm{C}$, for 5 to 7 days. Monosporic cultures were obtained from representative colonies and used for further testing.

\section{Identification of the isolates}

\section{Phenotypic characterization}

Moulds were subjected to a preliminary morphological identification. Spores of 7-day-old cultures on malt extract agar (MEA, HiMedia) were inoculated by 3-point inoculation in 9-cm Petri dishes containing MEA and Czapek Yeast Autolysate agar medium (CYA; HiMedia) and were analysed after incubation for 7 days in the dark, at $25^{\circ} \mathrm{C}$ for the following characters: colony size and texture, obverse and reverse colony colour, diffusible pigments and exudate production. The isolates were separated into 29 morphologically identical groups (morphotypes), and one representative isolate of each morphotype was used for molecular identification.

Isolates were screened for OTA production in coconut agar medium (CAM) as previously described (Heenan et al. 1998). For the isolates showing fluorescence on CAM, OTA production was confirmed by HLPC with fluorescence detection, following Vipotnik et al. (2017).

\section{Molecular identification}

Twenty-two isolates (representative of the morphotypes) selected for molecular identification were identified as follows. DNA was extracted by the SDS extraction protocol as described by Rodrigues et al. (2018). Moulds were identified by the ITS1/58S/ITS2 region of the rRNA gene (ca. $500 \mathrm{bp}$ ) using the primers ITS1-F/ITS4 (Gardes and Bruns 1993; White et al. 1990) or by a portion of the calmodulin gene ( $c m d A$; comprising exons 1 to 5 and introns 2 to 5 ; ca. $830 \mathrm{bp}$ ) using the primers CL1/CL2A (O'Donnell et al. 2000). PCR products were purified with the commercial kit GF-1 PCR CleanUp Kit (Vivantis) as described by the manufacturer.

Sequence analyses were carried out on an ABI 3730xl DNA Analyzer (Applied Biosystems). PCR products were sequenced in both directions, and a consensus sequence was assembled using the package Sequencher 4.9-Build 4751 (Gene Codes, Ann Arbor Michigan). The consensus sequences were adjusted using the programme BioEdit 
sequence alignment editor 7.0.5.1 and then aligned with the NCBI GenBank database (http://www.ncbi.nlm.nih.gov/) using the BLAST algorithm. Sequences were deposited in NCBI GenBank with the accession numbers MW940547 to MW940550 (ITS) and MW962279 to MW962295 (calmodulin) (Table S2).

\section{Detection and quantification of OTA in dates}

Ten grams of dates were homogenized in $20 \mathrm{~mL}$ of $70 \%$ methanol for $20 \mathrm{~min}$ in a magnetic stirrer. Five millilitres of filtered methanolic extract were collected into a $25-\mathrm{mL}$ measuring cylinder, and $20 \mathrm{~mL}$ of PBS-T (PBS/0.01\% Tween 80 ) were added and mixed well. The diluted extract was filtered with a microfibre glass filter and $20 \mathrm{~mL}$ of this extract $(=1 \mathrm{~g}$ sample) were passed through OchraTest ${ }^{\mathrm{TM}}$ immunoaffinity column (IAC). The column was then washed with $10 \mathrm{~mL}$ of PBS-T, followed by $10 \mathrm{~mL}$ ultra-pure water. OTA was eluted with $1.5 \mathrm{~mL}$ HPLC-grade methanol with $2 \%$ acetic acid (98:2; v:v). OTA was quantified by HPLC with fluorescence detection, as previously described (Vipotnik et al. 2017).

For method validation, recovery rates, limit of detection (LOD), limit of quantification (LOQ) and linearity were determined taking into account the harmonised guidelines for in-house method validation presented in the Commission Regulation (EC) No 401/2006 (EC 2006b). The OTA working solution $(\mathrm{C}=1000 \mathrm{ng} / \mathrm{mL})$ was prepared in methanol $(\mathrm{MeOH})$ using commercial standard OTA (Sigma) and stored at $-20{ }^{\circ} \mathrm{C}$ until use. Linearity, LOD and LOQ were determined by three series of analyses, using 11 standard solutions with concentrations ranging from 0.05 to $100 \mathrm{ng} /$ $\mathrm{mL}$. LOD and LOQ were calculated according to Taverniers et al. (2004). For recovery tests, the extraction procedure previously described was applied to dates spiked with $10 \mathrm{ng}$ of OTA per gram of sample. Spiked samples were left at $4{ }^{\circ} \mathrm{C}$ overnight for toxin incorporation previously to extraction. This procedure was made in triplicate. Recovery rates of OTA were calculated as the ratio of recovered mycotoxin concentration relative to the spiked concentration. Precision was calculated in terms of intra-day repeatability $(n=3)$.

\section{Statistical analysis}

Fungal occurrence was determined as the percentage of samples contaminated with a given species. Fungal frequency was determined as the percentage of isolates of a given species compared with the total number of isolates. The effect of variety, origin, format, packaging and preservatives on the total fungal counts and Aspergillus sect. Nigri counts was assessed by a generalized linear model following a Poisson distribution and log link function. The analysis was developed using the IBM SPSS Statistics version 22.

\section{Results and discussion}

\section{Mycobiota profile of date samples}

The total number of fungal isolates was 70 , obtained from 10 out of 19 samples. No isolates were obtained from samples S01, S02, S03, S06, S07, S08, S15, S16 and S17. Twenty-one isolates were identified from the 29 morphotype representative isolates (Table S2). The remaining 8 isolates were identified as Aspergillus nigerl Aspergillus tubingensis but were not confidently discriminated by the calmodulin gene sequence. Aspergillus niger aggregate is further used in this study to refer to this group of isolates identified as A. niger/A. tubingensis.

Table 1 shows the results for total fungal counts, total Aspergillus sect. Nigri counts and occurrence of each species in the samples. In the present study, only Aspergillus and Penicillium genera were detected in the samples. Aspergillus species consisted of $83 \%$ of the total isolates, while Penicillium species accounted for $17 \%$. Aspergillus section Nigri isolates were the most common in the Aspergillus genus ( $70 \%$ of total isolated fungi, occurring in $42 \%$ of the samples), while A. flavus represented $10 \%$ of total isolates, with $26 \%$ occurrence. Penicillium citrinum was the most frequently isolated species from the genus Penicillium (10\% of total isolates) but was detected in only 2 samples. The remaining species were rarely isolated. Previous studies on fungal contamination of palm dates report similar results (Abdel-Sater and Saber 1999; Azaiez et al. 2015; Gherbawy et al. 2012; Nass 2017; Quaglia et al. 2020). Aspergillus section Nigri are distributed worldwide and are regarded as common food spoilage fungi (Ismail 2017; Cabañes and Bragulat 2018), and A. tubingensis has been frequently reported in Mediterranean countries (Pantelides et al. 2017; Gil-Serna et al. 2019; Quaglia et al. 2020).

As presented in Table 1, most samples showed low or undetectable fungal loads, while three samples (S04, S12 and S13) had a significantly higher frequency of contamination for both fungal counts and Aspergillus section Nigri $(p=0.000)$. Figure 1 shows the difference in fungal diversity and cumulative number of isolates (average/sample) in samples with and without chemical preservation. As set in the samples' label (Table S1), those three samples were labelled as bioproducts or extra-quality, with no chemical additives, and the remaining was treated with chemical preservatives and stabilisers such as sorbitol (E420ii), sulphites, benzoic acid, citric acid and potassium sorbate. The absence of preservatives significantly influenced the fungal counts, but no significant differences were registered between the different types of preservatives ( $p=0.000$; data not shown).

The relatively low contamination levels reported in the analysed samples are probably due to the strict regulations 


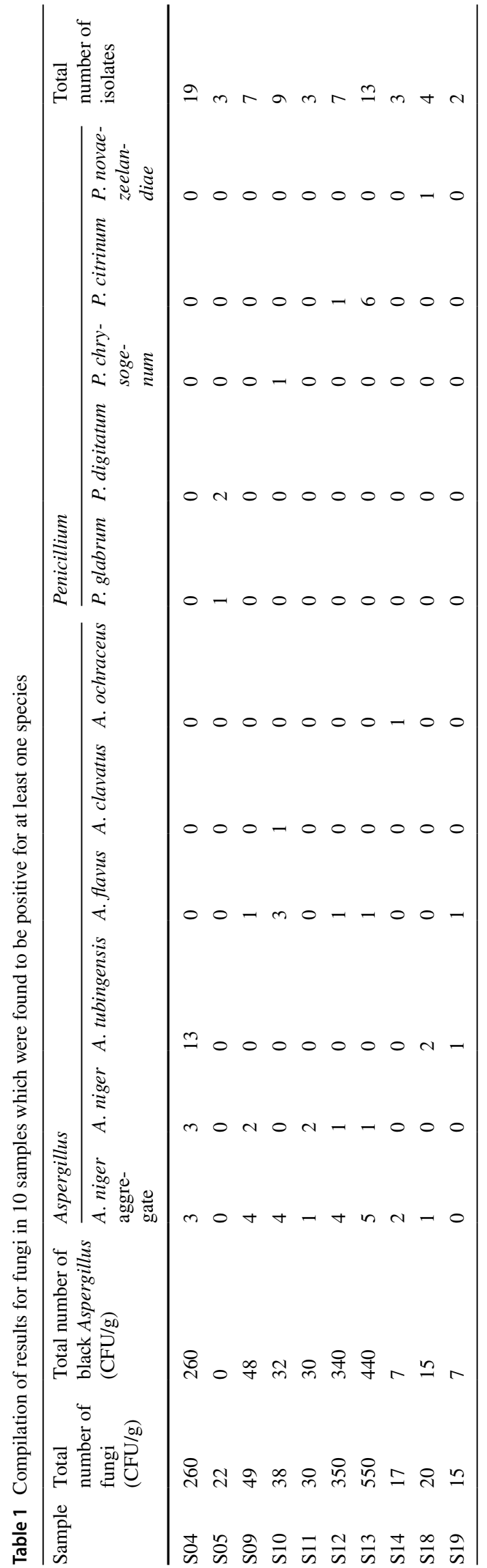

in the European Union, which prevent highly contaminated samples from entering the markets.

Considering the mycotoxigenic profile of the isolated fungi, from the 46 isolates tested in CAM, only P. citrinum and Aspergillus ochraceus showed a green fluorescence under UV light. These two isolates as well as seven isolates representative of $A$. niger aggregate (who tested negative for OTA on CAM) were also tested by HPLC to confirm the ochratoxigenic profile. Only A. ochraceus was confirmed to produce OTA, whereas all A. niger aggregate isolates confirmed to be negative. This result is in agreement with previous studies reporting that $A$. tubingensis is a non-ochratoxigenic species, while only few isolates of $A$. niger are able to produce OTA (Abarca et al. 2004; Gil-Serna et al. 2019; Quaglia et al. 2020). Aspergillus flavus were also considered non-aflatoxigenic (only screened on CAM). These results are lower than others previously reported. Gherbawy et al. (2012) reported that 18 out of 80 A. flavus isolates (39\%) were aflatoxigenic, while nine out of 36 isolates of A. niger (25\%) were ochratoxigenic. Quaglia et al. (2020) reported that the only A. flavus isolated from dried dates was able to produce aflatoxins B1 and $\mathrm{B} 2$, while none of the $16 \mathrm{~A}$. tubingensis produced OTA.

\section{OTA contamination of date samples}

The method of extraction and quantification of OTA in palm dates was validated with high precision $\left(y=820002 x+444,102 ; r^{2}=0.998\right)$. The recovery rate of OTA in spiked date samples at a concentration of $10 \mu \mathrm{g} / \mathrm{kg}$ was $94.8 \%$, with RSDr of 0.9. LOD and LOQ were calculated as $0.17 \mathrm{ng} / \mathrm{mL}$ and $0.58 \mathrm{ng} / \mathrm{mL}$, respectively. From the 19 samples analysed for OTA contamination, only sample S12 (no additives, extra-quality) showed detectable levels of OTA, at a concentration of $0.75 \mu \mathrm{g} / \mathrm{kg}$. Although no maximum tolerable limit of OTA has been established for dried dates, this value is under the limit set for dried grape fruits $(10 \mu \mathrm{g} / \mathrm{kg}$ ) set by the European Commission (EC 2006a).

Iamanaka et al. (2005) observed OTA contamination up to $5 \mu \mathrm{g} / \mathrm{kg}$ in 2 out of 20 dried date samples sold in Brazil. Azaiez et al. (2015) reported that OTA was detected in 38\% of the Tunisian date samples (mean $1.26 \mu \mathrm{g} / \mathrm{kg}$ ), with the highest detected level of $3.34 \mu \mathrm{g} / \mathrm{kg}$. This study revealed a higher mycotoxin incidence in date samples collected from Tunisia in comparison to those collected from Spain, although more than $80 \%$ of the latter were imported from Tunisian markets (Deglet Nour variety). This could be due to the samples purchased in Tunisia having different quality classes, as some of them belonged to low quality categories and were intended for use as feed. Indeed, the contamination profile of the dates belonging to the variety "Deglet Nour" purchased in Tunisia was similar to those purchased in Spain (Azaiez et al. 2015). 
Fig. 1 Cumulative number of isolates of each fungal species, in average, in dried dates with and without preservatives

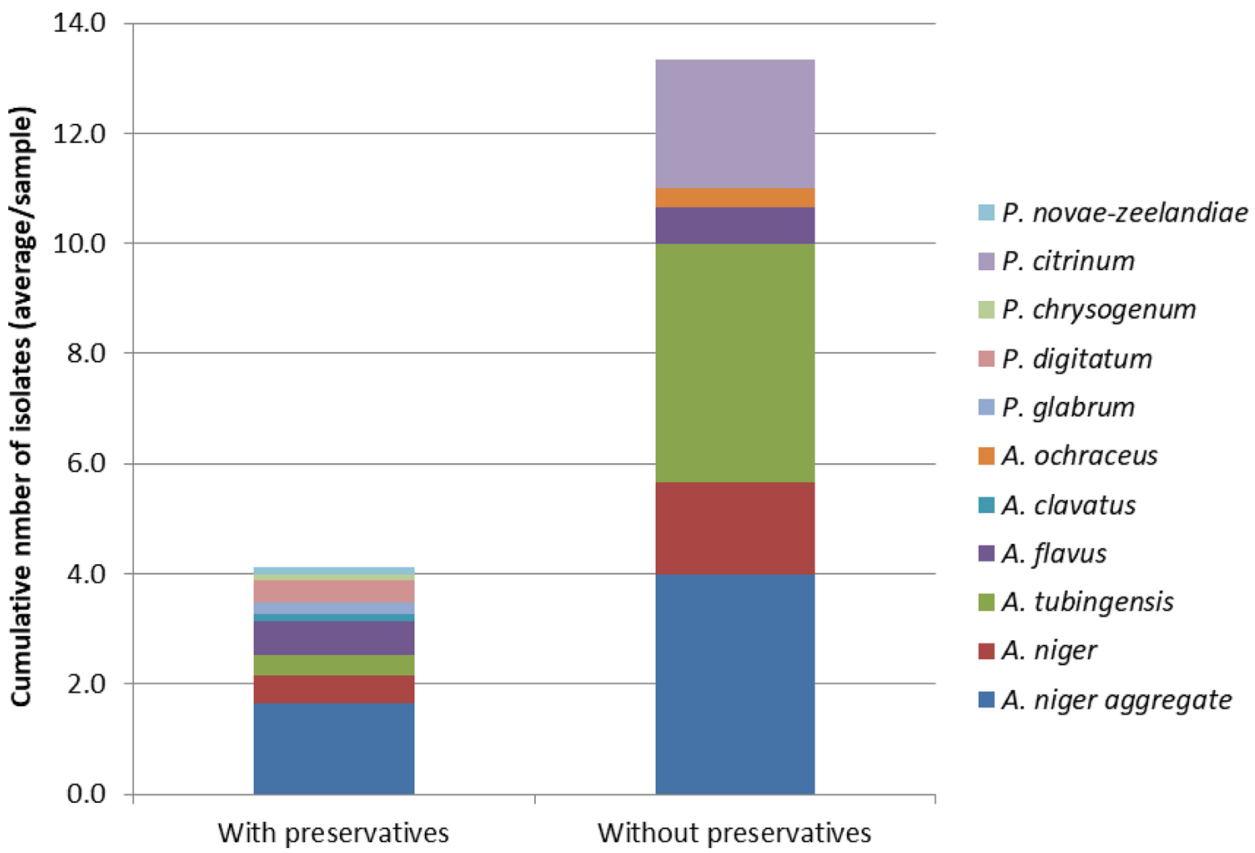

Han et al. (2016) revealed that $22.5 \%$ of date samples were contaminated with OTA with levels as high as $213 \mu \mathrm{g} /$ $\mathrm{kg}$, while Abdallah et al. (2018) detected OTA in 3 samples (11\%) with a contamination ranging from as low as $1.5 \mu \mathrm{g} /$ $\mathrm{kg}$ to the extremely high level of $6070 \mu \mathrm{g} / \mathrm{kg}$, suggesting that the OTA-producing fungi, in particular Aspergillus species, are able to produce high amounts of OTA in these dried fruits. The low percentage and level of OTA contamination in date samples observed in the current study may be explained with the low contamination rate with mycotoxigenic fungi or can be a result of the use of chemical preservatives.

Recently, EFSA estimated that the mean daily dietary exposure (ng/kg bw per day) of the European populations to OTA varies between 1.88 and $5.72 \mathrm{ng} / \mathrm{kg}$ bw per day (lower bound) and between 3.81 and $12.33 \mathrm{ng} / \mathrm{kg}$ bw per day (upper bound) EFSA 2020). Dried fruits are among the groups of foods with the highest contribution to this exposure, and dates are third in the list, only after dried figs and vine fruits (EFSA 2020). Considering the low OTA contamination levels (in both occurrence and amount) of the analysed samples, and the apparently low consumption of this fruit by the Portuguese population (FAOSTAT 2021; Ribeiro and Fernandes 2017), the exposure to OTA seems to be negligible. Even so, the importation and consumption of dried dates are steadily increasing.in Portugal (FAOSTAT 2021).

In conclusion, commercial dried date fruits from Portuguese markets with different origins and brands seem to be safe in terms of ochratoxigenic fungi and OTA contamination. These results can be due to the strict regulations set for the EU markets, which reduce the probability of highly contaminated samples entering the markets. The highest incidence of fungal contamination was observed for extraquality or biological products, without chemical preservatives. Even though the high fungal contamination did not generally reflect in detectable OTA contamination, this can be due to the fact that only one OTA-producing fungus was detected. In this respect, it will be necessary to further study the influence of the chemical preservatives on fungal growth and OTA production in dates and to develop biological strategies to reduce the need for these chemical additives.

Supplementary information The online version contains supplementary material available at https://doi.org/10.1007/s12550-021-00432-0.

Author contribution Conceptualization: Paula Rodrigues; Methodology: Paula Rodrigues; Formal analysis and investigation: Iva Nikolchina; Writing —original draft preparation: Iva Nikolchina; Writing—review and editing: Paula Rodrigues; Funding acquisition: Paula Rodrigues; Resources: Paula Rodrigues; Supervision: Paula Rodrigues.

Funding This work was supported by the Foundation for Science and Technology (FCT, Portugal) and FEDER under Programme PT2020 for financial support to CIMO (UID/AGR/00690/2019).

\section{References}

Abarca ML, Accensi F, Cano J, Cabañes FJ (2004) Taxonomy and significance of black aspergilli. ALJMAO 86:33-49

Abdallah M, Krska R, Sulyok M (2018) Occurrence of ochratoxins, fumonisin B2, aflatoxins (B1 and B2), and other secondary fungal metabolites in dried date palm fruits from Egypt: a mini-survey. J Food Sci 83:559-564 
Abdel-Sater MA, Saber SM (1999) Mycoflora and mycotoxins of some Egyptian dried fruits. Bull Fac Scient Assiut Univer 28:91-107

Aksoy U, Eltem R, Meyvaci KB, Altindisli A, Karabat S (2007) Fiveyear survey of ochratoxin A in processed sultanas from Turkey. Food Addit Contam 24:292-296

Al-Shahib W, Marshall RJ (2003) The fruit of the date palm: its possible use as the best food for the future? Int J Food Sci Nutr $54: 247-259$

Azaiez I, Font G, Mañes J, Fernández-Franzón M (2015) Survey of mycotoxins in dates and dried fruits from Tunisian and Spanish markets. Food Control 51:340-346

Bircan C, Koç M (2012) Aflatoxins in dried figs in Turkey: a comparative survey on the exported and locally consumed dried figs for assessment of exposure. J Agric Sci Technol 14:1265-1274

Cabañes FJ, Bragulat MR (2018) Black aspergilli and ochratoxin-A producing species. Curr Opin Food Sci 23:1-10

Calcagni P (2018) Sustainable production and trade of nuts and dried fruit: general overview. UNECE-FAO symposium: nuts and dried fruit for a sustainable future. Geneva, June 26, 2018

CBI (Centre for the Promotion of Imports from developing countries) (2020) The European market potential for dates. CBI Market Information Database. https://www.cbi.eu/market-information/ processed-fruit-vegetables-edible-nuts/dates/market-potential. Accessed 25 March 2021

European Commission (2006a) Commission Regulation (EC) No 1881/2006 of 19 December 2006 Setting maximum levels for certain contaminants in foodstuffs. Off J Eur Union L364:5-24.L. Last consolidated version available from: https://eur-lex.europa. eu/legal-content/DE/AUTO/?uri=CELEX:02006R1881-20180319

European Commission (2006b) Commission Regulation (EC) No 401/2006 of 23 February 2006 laying down the methods of sampling and analysis for the official control of the levels of mycotoxins in foodstuffs. Off J Eur Union L70: 12-34. Last consolidated version available from: https://eur-lex.europa.eu/legal-content/EN/ TXT/PDF/?uri=CELEX:02006R0401-20140701

European Food Safety Authority, Panel on Contaminants in the Food Chain (2020) European food safety authority (EFSA) scientific opinion on risk assessment of ochratoxin A in food. EFSA J 18(5):6113. Available from:https://efsa.onlinelibrary.wiley.com/ doi/10.2903/j.efsa.2020.6113

Food and Agriculture Organization of the United Nations Statistics (FAOSTAT) (2021) http://www.fao.org/faostat/en/\#data/FBS. Accessed 28 March 2021

Gardes M, Bruns TD (1993) ITS primers with enhanced specificity for Basidiomycetes: application to identification of mycorrhizae and rusts. Mol Ecol 2:113-118

Gherbawy YA, Elhariry HM, Bahobial AAS (2012) Mycobiota and mycotoxins (aflatoxins and ochratoxin) associated with some Saudi date palm fruits. Foodborne Pathog Dis 9:561-567

Gil-Serna J, García-Díaz M, Vázquez C, González-Jaén MT, Patiño B (2019) Significance of Aspergillus niger aggregate species as contaminants of food products in Spain regarding their occurrence and their ability to produce mycotoxins. Food Microbiol 82:240-248

Han Z, Dong M, Han W, Shen Y, Nie D, Shi W, Zhao Z (2016) Occurrence and exposure assessment of multiple mycotoxins in dried fruits based on liquid chromatography-tandem mass spectrometry. World Mycotox J 9:465-474

Heenan CN, Shaw KJ, Pitt JI (1998) Ochratoxin A production by Aspergillus carbonarius and A. niger and detection using coconut cream agar. J Food Mycol 1:67-72

Heperkan D, Moretti A, Dikmen CD, Logrieco AF (2012) Toxigenic fungi and mycotoxin associated with figs in the Mediterranean area. Phytopathol Mediterr 51:119-130
Heshmati A, Zohrevand T, Mousavi A, Sasan A, Nejad M, Sant'Ana AS, (2017) Co-occurrence of aflatoxins and ochratoxin A in dried fruits in Iran: dietary exposure risk assessment. Food Chem Toxicol 106:202-208

Iamanaka BT, Taniwaki MH, Menezes HC, Vicente E, Fungaro MHP (2005) Incidence of toxigenic fungi and ochratoxin A in dried fruits sold in Brazil. Food Addit Contam 22:1258-1263

Iamanaka BT, de Menezes HC, Vicente E, Leite RSF, Taniwaki MH (2007) Aflatoxigenic fungi and aflatoxins occurrence in sultanas and dried figs commercialized in Brazil. Food Control 18:454-457

Ismail MA (2017) Incidence and significance of black aspergilli in agricultural commodities: a review, with a key to all species accepted to-date. Eur J Biol Res 7:207-222

Nass LA (2017) Fungal contamination and invertase activity in dates and date products in Saudi Arabia. Amer J Food Technol 12:295-300

O’Donnell K, Nirenberg HI, Aoki T, Cigelnik E (2000) A multigene phylogeny of the Gibberella fujikuroi species complex: detection of additional phylogenetically distinct species. Mycosci 41:61-78

Ozer H, Oktay Basegmez HI, Ozay G (2012) Mycotoxin risks and toxigenic fungi in date, prune and dried apricot among Mediterranean crops. Phytopathol Mediterr 51:148-157

Pantelides IS, Aristeidou E, Lazari M, Tsolakidou MD, Tsaltas D, Christofidou M, Kafouris D, Christou E (2017) Biodiversity and ochratoxin A profile of Aspergillus section Nigri populations isolated from wine grapes in Cyprus vineyards. Food Microbiol 67:106-117

Quaglia M, Santinelli M, Sulyok M, Onofri A, Covarelli L, Beccari G (2020) Aspergillus, Penicillium and Cladosporium species associated with dried date fruits collected in the Perugia (Umbria, Central Italy) market. Int J Food Microbiol 322:108585

Rahimi E, Shakerian A (2013) Ochratoxin A in dried figs, raisings, apricots, dates on Iranian retail market. Health 5:2077-2080

Ribeiro MI, Fernandes A (2017) Consumo e hábitos de compra de fruta desidratada no Norte de Portugal. 2nd Congress of the Agrarian Schools, 16-18 November (poster) http://hdl.handle.net/10198/ 14728

Rodrigues P, Venâncio A, Lima N (2018) Toxic reagents and expensive equipment: are they really necessary for the extraction of good quality fungal DNA? Lett Appl Microbiol 66:32-37

Shenasi M, Aidoo KE, Candlish AAG (2002a) Microflora of date fruits and production of aflatoxins at various stages of maturation. Int $\mathrm{J}$ Food Microbiol 79:113-119

Shenasi M, Candlish AAG, Aidoo KE (2002b) The production of aflatoxins in fresh date fruits and under simulated storage conditions. J Sci Food Agri 82:848-853

Taverniers I, de Loose M, Van Bockstaele E (2004) Trends in quality in the analytical laboratory. II. Analytical method validation and quality assurance. Trends Anal Chem 23:535-552

Vipotnik Z, Rodríguez A, Rodrigues P (2017) Aspergillus westerdijkiae as a major ochratoxin A risk in dry-cured ham based-media. Int $\mathrm{J}$ Food Microbiol 241:244-251

White TJ, Burns T, Lee S, Taylor JW (1990) Amplification and direct sequencing of fungal ribosomal DNA for phylogenetics. In: Inns MA, Gelfand DH, Sninsky JJ, White TJ (eds) PCR protocols: a guide to methods and applications. Academic Press, San Diego, CA, USA, pp 315-321

Zaid A, de Wet PF (2002) Chapter II: origin, geographical distribution and nutritional values of date palm. In: Zaid A (ed) Date palm cultivation. FAO Plant Production and Protection Paper 156 Rev. 1, Rome, Italy. ISSN 0259-2517

Publisher's Note Springer Nature remains neutral with regard to jurisdictional claims in published maps and institutional affiliations. 